\title{
Electron Partitioning During Light- and Nutrient-Powered Hydrogen Production by Rhodobacter sphaeroides
}

\author{
Luftu Safak Yilmaz • Wayne S. Kontur • \\ Alison P. Sanders • Ugur Sohmen • \\ Timothy J. Donohue • Daniel R. Noguera
}

Published online: 21 January 2010

(C) Springer Science+Business Media, LLC. 2010

\begin{abstract}
The partitioning of reducing power into different electron-accepting pathways was evaluated during growth and stationary phases of $\mathrm{H}_{2}$-producing Rhodobacter sphaeroides cultures. For this, an electron balance method was developed using the chemical oxygen demand concept to quantitatively analyze the partitioning of nutrient electrons into $\mathrm{H}_{2}$, cell biomass, polyhydroxybutyrate (PHB), and soluble microbial products (SMP). Overall, these four electron sinks were accounted for greater than $85 \%$ of the electrons provided by the nutrients. Glucose, lactate, succinate, fumarate, and pyruvate were individually provided as the main carbon source, and in all cases, glutamate was provided as a nitrogen source in order to enhance $\mathrm{H}_{2}$ production. About $25-35 \%$ of the electrons ended up in $\mathrm{H}_{2}$ during growth, while up to $60 \%$ of the electrons partitioned into $\mathrm{H}_{2}$ in some stationary phase cultures. The other two major electron sinks in the growth phase were cell mass and PHB, while in stationary phase, SMP were accounted for $>30 \%$ of the substrate electrons utilized. In general, the largest portion of SMP comprised
\end{abstract}

Submission as a research paper to BioEnergy Research

L. S. Yilmaz • A. P. Sanders • U. Sohmen • D. R. Noguera $(\bowtie)$

Department of Civil and Environmental Engineering,

University of Wisconsin-Madison,

3216 Engineering Hall, 1415 Engineering Drive,

Madison, WI 53706, USA

e-mail: noguera@engr.wisc.edu

W. S. Kontur · T. J. Donohue

Department of Bacteriology, University of Wisconsin,

Madison, WI, USA

L. S. Yilmaz - W. S. Kontur · T. J. Donohue - D. R. Noguera Great Lakes Bioenergy Research Center, University of Wisconsin, Madison, WI, USA low-molecular weight $(<3 \mathrm{kDa})$ compounds mostly produced during stationary phase, although larger-size molecules were also detected in both phases. Overall, the fractions of electrons that partitioned into $\mathrm{H}_{2}(0.21$ to 0.35 ) and PHB (0.06 to 0.21$)$ were highly correlated with the standard free energy change of the substrate oxidation half-reaction equation, normalized per electron equivalent. In a $\mathrm{PHB}(-)$ mutant, electron redistribution increased $\mathrm{H}_{2}$ production, the extent of which depended on the carbon source provided.

Keywords Biofuel · Hydrogen - Electron balance . PHB mutant $\cdot$ Rhodobacter sphaeroides

$\begin{array}{ll}\text { Abbreviations } \\ \text { BAME } & \text { Benzoic acid methyl ester } \\ \text { COD } & \text { Chemical oxygen demand } \\ \text { eob } & \text { End of batch } \\ \text { EPS } & \text { Extracellular polymeric substances } \\ \text { FID } & \text { Flame ionization detector } \\ \text { Fum } & \text { Fumarate } \\ \text { Glc } & \text { Glucose } \\ \text { Lac } & \text { Lactate } \\ \text { me } & \text { Midexponential point } \\ \text { mst } & \text { Midstationary point } \\ \text { pe } & \text { Beginning of postexponential phase } \\ \text { PEGP } & \text { Postexponential growth phase } \\ \text { PHB } & \text { Polyhydroxybutyrate } \\ \text { Pyr } & \text { Pyruvate } \\ \text { SMP } & \text { Soluble microbial products } \\ \text { st } & \text { Beginning of stationary phase } \\ \text { STP } & \text { Stationary phase } \\ \text { Suc } & \text { Succinate } \\ \text { WT } & \text { Wild type }\end{array}$




\section{Introduction}

Among the biologically based fuels that are being considered or developed as renewable replacements for fossil fuels are ethanol, various next generation liquid transportation additives, or gases like $\mathrm{H}_{2}$ or $\mathrm{CH}_{4}$ [1]. Strategies to achieve efficient biological routes for the production of these fuels require not only an understanding of the pathways by which cells partition the carbon skeletons and reducing power needed for fuel production, but also knowledge about the competing processes that detract from biofuel production. Using $\mathrm{H}_{2}$ as a model biofuel, we are interested in determining how cells partition available reducing power into different electron-accepting pathways. The goal of these studies is to identify competing pathways that can potentially be eliminated to optimize $\mathrm{H}_{2}$ production.

Biologically based $\mathrm{H}_{2}$-producing systems have the potential to reduce the amount of fossil fuels used for the fertilizer, chemical, petroleum, or steel industries, since $\mathrm{H}_{2}$ synthesis for their use accounts for approximately $10 \%$ of the national fossil fuel use [2]. Moreover, about $95 \%$ of the current global $\mathrm{H}_{2}$ is produced by individual industries to satisfy their own needs [3]. Such a decentralized production approach is an attractive model for the implementation of biological $\mathrm{H}_{2}$ production technologies.

The biological sources of $\mathrm{H}_{2}$ currently under consideration include both fermentative and photosynthetic organisms [4, 5]. Among the photosynthetic microbes already known to produce $\mathrm{H}_{2}$ are photoheterotrophic bacteria, which have the ability to simultaneously use energy derived from light and organic compounds to drive $\mathrm{H}_{2}$ production [6], and therefore, can be used to develop solar-driven biofuel production processes for the utilization of renewable organic substrates in a biofuel economy. In addition, the potential for recycling organic waste into $\mathrm{H}_{2}$ via photoheterotrophic metabolism [7, 8] or a combination of dark and light fermentations [9-11] has also been advocated, potentially leading to on-site reutilization of organic wastes by industries that currently use $\mathrm{H}_{2}$ produced from nonrenewable resources.

In this study, we quantitatively determined the fate of electrons in $\mathrm{H}_{2}$-producing batch cultures of the purple nonsulfur bacterium Rhodobacter sphaeroides during growth and stationary phases. $R$. sphaeroides is known to catalyze light- and nutrient-powered $\mathrm{H}_{2}$ production at significant rates [12-14]. Past studies of $\mathrm{H}_{2}$ production by this and other related bacteria have implicated nitrogenase activity as a source of this compound $[6,8,15]$. In addition, genetic and genomic techniques are available [16-18] and can be used to test predictive models for how individual pathways impact the flow of reducing power to $\mathrm{H}_{2}$. Based on prior knowledge on $R$. sphaeroides and other photoheterotrophic organisms $[8,19]$, a conceptual model of electron partitioning into pathways that contribute to and detract from $\mathrm{H}_{2}$ production is available (Fig. 1). Thus, our work sought to develop methods to assess the partitioning of reducing power into $\mathrm{H}_{2}$ production when wild type or mutant strains were provided light and different organic substrates.

Our approach is based on the theoretical or experimental assessment of the chemical oxygen demand (COD) of products from four electron-accepting reactions (Fig. 1). These products include new cells (biomass), polyhydroxybutyrate (PHB), soluble microbial products (SMP), and $\mathrm{H}_{2}$. Since COD is indicative of the reduction state of each product [20], this method allowed the quantitative determination of electron fate in a mass balance model (Fig. 1).

\section{Methods}

\section{Bacterial Strains}

All experiments were carried out using wild-type $R$. sphaeroides 2.4.1. or its derivative $\Delta 0382$, which is deficient in PHB synthesis. To construct $\Delta 0382$, a $5.4-\mathrm{kb}$ fragment containing RSP0382 (annotated as coding for PHB synthase) plus $1.8-\mathrm{kb}$ upstream and $1.7-\mathrm{kb}$ downstream flanking DNA was amplified from the genome of strain 2.4.1 via polymerase chain reaction (PCR) using the upstream primer 5'-ACTCTAGACCTACGATTTCATG CCCGA-3' (containing an XbaI restriction site, underlined) and the downstream primer $5^{\prime}$-TAGCATGCCGTCCGACT ATTCCGAAG-3' (containing an SphI restriction site, underlined). This fragment was digested with $\mathrm{XbaI}$ and $\mathrm{SphI}$ and cloned into XbaI- and SphI-digested pUC19 to generate plasmid pUC19/RSP0382. PCR amplification of this plasmid using the primers 5'-ATAGATCTTCGACCGACAAGAC CTTCATTC- 3 ' and 5'-ATAGATCTTGCTCTTCGGTTGCC ATATTGAC-3' (both containing BgIII restriction sites, underlined) was used to generate a 1,491-bp in-frame deletion within RSP0382. The resulting DNA fragment was digested with BglII, and then treated with T4 DNA ligase to produce plasmid $p U C 19 / \Delta 0382$. This plasmid was digested with XbaI and SphI, and purified DNA containing the deletion in RSP0382 was cloned into XbaI- and SphIdigested pK18mobsacB (which contains kanamycin resistance and sucrose sensitivity ( $\mathrm{sacB}$ ) genes) to produce $\mathrm{pK} 18 \mathrm{mobsacB} / \Delta 0382$. This plasmid was transformed into Escherichia coli S17-1 and mobilized via conjugation into $R$. sphaeroides 2.4.1. Kanamycin-resistant, sucrose-sensitive exconjugants, in which the plasmid was incorporated into the genome via homologous recombination, were isolated, and then grown in medium lacking kanamycin and sucrose. Sucrose-resistant, kanamycin-sensitive cells, in which the plasmid was excised via a second homologous recombina- 
Fig. 1 Electron fate model of $R$. sphaeroides. Fraction of substrate (organic carbon and nitrogen source) electrons is designated by $f$. Inset equation shows electron mass balance

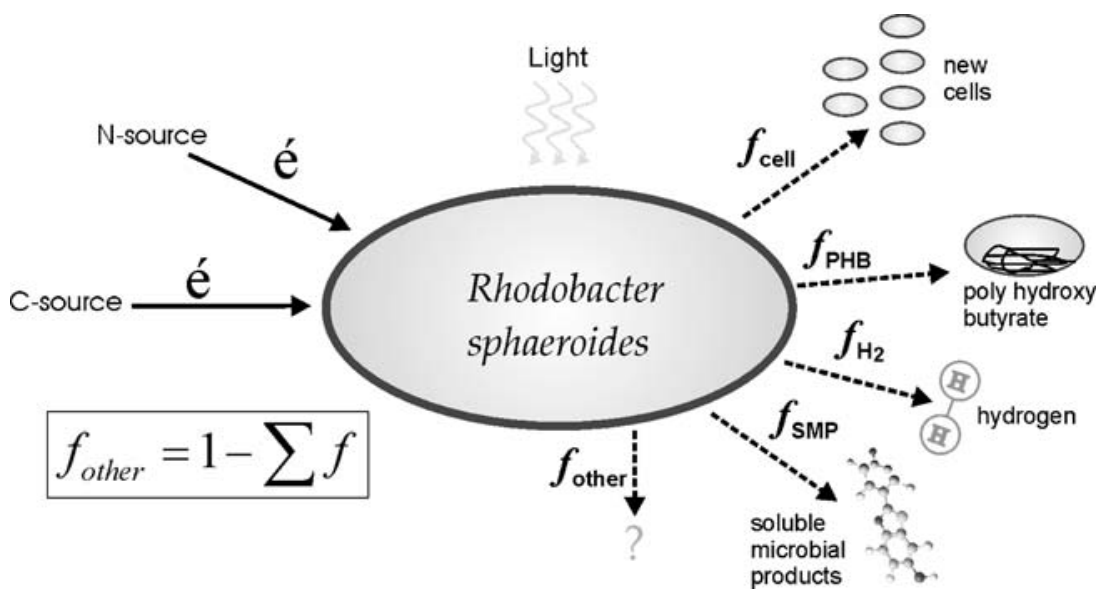

tion event, were isolated and screened by PCR for the deletion within RSP0382.

\section{Growth Conditions and Sampling}

Cultures were first grown aerobically in Sistrom's minimal medium [21] amended with $33.9 \mathrm{mM}$ succinate as carbon source and ammonia as nitrogen source, and then under anaerobic photosynthetic conditions in a modified Sistrom's medium with $8.1 \mathrm{mM}$ glutamate as the only nitrogen source and one of the following carbon sources: $39.5 \mathrm{mM}$ lactate, $33.9 \mathrm{mM}$ succinate, $39.5 \mathrm{mM}$ fumarate, $47.4 \mathrm{mM}$ pyruvate, or $19.8 \mathrm{mM}$ glucose (concentrations were determined to meet a targeted constant COD of $3,800 \mathrm{mg} / \mathrm{L}$ for the carbon source). Cultures to be analyzed were inoculated by transferring $200 \mu \mathrm{L}$ of photosynthetically grown cells into test tubes $(\sim 21 \mathrm{~mL}$ total volume) with $20 \mathrm{~mL}$ of fresh medium containing the same carbon source.

Photosynthetic cells were grown at $30^{\circ} \mathrm{C}$ continuously illuminated by an incandescent light source that produced approximately $10 \mathrm{~W} / \mathrm{m}^{2}$, as measured with a YellowSprings-Kettering model 6.5-A radiometer through a Corning 7-69 filter. Cell turbidity was monitored using a Klett-Summerson photoelectric colorimeter (Klett MFG Co., NY). Turbidity readings were converted to colonyforming units per milliliter using previously described conversion factors for photosynthetic cultures [22].

To measure the amount of gas produced, cultures were capped gastight with a rubber septum and the $1-\mathrm{mL}$ headspace connected to an AER-200 respirometer (Challenge Technology, Springdale, AR) via a needle ( $\geq 20 \mathrm{G}$ ) and either 3/32-in. Tygon FTM tubing provided by the manufacturer or $2.06-\mathrm{mm}$ Masterflex Viton extension tubing (Cole-Parmer, Vernon Hills, IL). The data acquisition and control software for the respirometer was set to anaerobic mode, during which, the number of bubbles passing through the unit was recorded in real time and converted to volume (milliliter) using factoryderived calibration constants.
Two or three cultures (biological replicates) were sampled at different phases of growth and gas production. For the analysis of gas composition, $\sim 3-7 \mathrm{~mL}$ of gas was removed from the culture headspace. Subsequently, $2 \mathrm{~mL}$ of culture was sampled and immediately frozen for total COD analysis, while the remaining culture was centrifuged $\left(6,000 \mathrm{rpm}, 12 \mathrm{~min}, 4^{\circ} \mathrm{C}\right)$. The supernatant was filtered through $0.2-\mu \mathrm{m}$ syringe filters, and the filtered supernatant and cell pellets were immediately frozen and stored for analysis of SMP and PHB, respectively. When indicated, selected supernatants were passed through prewashed $10-$ or $3-\mathrm{kDa}$ molecular weight cutoff filters (Millipore Microcon, Bedford, MA) for SMP size distribution analysis.

\section{Analytical Methods}

The gas composition of headspace samples was analyzed using a GC-2014 gas chromatograph (Shimadzu Scientific, Columbia, MD). A flame ionization detector (FID) (set at $\left.200^{\circ} \mathrm{C}\right)$ coupled to a methanizer $\left(380^{\circ} \mathrm{C}\right)$ and a thermal conductivity detector $\left(100^{\circ} \mathrm{C}\right)$ were used for measuring $\mathrm{CO}_{2}$ and $\mathrm{H}_{2}$, respectively. The column oven temperature was $60^{\circ} \mathrm{C}$. Argon was used as the carrier gas. For quantification, a standard of $80 \% \mathrm{H}_{2}$ and $20 \% \mathrm{CO}_{2}$ (Airgas, Carol Stream, IL) and a Scotty 48 standard (Scotty Specialty Gases, Plumsteadville, PA) were used. The FID-methanizer system was capable of detecting volatile organic compounds such as ethylene, formic acid, and ethanol, although no significant amounts of these compounds were observed from the culture headspace samples tested (data not shown). Peaks for $\mathrm{H}_{2}, \mathrm{CO}_{2}, \mathrm{O}_{2}$, and $\mathrm{N}_{2}$ gases were typically detected, with the latter two being derived from small amounts of air introduced during the processing of samples from the cultures. A correction was therefore applied to calculate the $\mathrm{H}_{2}$ partial pressure in the cultures by removing the contributions of $\mathrm{O}_{2}$ and $\mathrm{N}_{2}$. The amount of $\mathrm{H}_{2}$ produced was calculated as the average $\mathrm{H}_{2}$ 
partial pressure during a batch times the total gas volume quantified by the respirometer.

Organic acids in culture supernatants were quantified by gas chromatography-mass spectrometry (GC-MS; GC-2010 gas chromatograph coupled to a QP-2010S mass spectrometer detector; Shimadzu Scientific) after derivatization with methyl chloroformate [23] and using benzoic acid methyl ester (BAME) as an internal standard. The carrier gas was helium, and the GC column was a SHRXI-5MS (Shimadzu Scientific). The temperatures for column oven, injection, ion source, and interface were set at $60^{\circ} \mathrm{C}, 180^{\circ} \mathrm{C}, 220^{\circ} \mathrm{C}$, and $275^{\circ} \mathrm{C}$, respectively. Standards of known concentrations were used to generate calibration curves for the quantification of identified peaks.

Sugar analysis was performed by high-performance liquid chromatography (model 10A-vP equipped with an RID-10A refractive index detector, Shimadzu Scientific) using operational settings established by the National Renewable Energy Laboratory [24]. Separation was achieved with an HPX-87P column (Biorad, Hercules, CA) set at $80^{\circ} \mathrm{C}$, and detection was by refractive index at the same temperature. The mobile phase was $0.005 \mathrm{M}$ $\mathrm{H}_{2} \mathrm{SO}_{4}$. Sugar standards were used for the generation of standard curves.

The PHB content of cells was determined following established procedures $[25,26]$ with slight modifications. Briefly, freeze-dried cell pellets were weighed, digested overnight in acidified methanol and chloroform at $105^{\circ} \mathrm{C}$, mixed with deionized water to induce phase separation, and analyzed by GC-MS. Column oven and injection temperatures were set at $50^{\circ} \mathrm{C}$ and $210^{\circ} \mathrm{C}$, respectively. PHB quantification was based on a PHB standard (Sigma-Aldrich, Steinheim, Germany) and with BAME as an internal standard. The fraction of PHB in biomass was calculated based on the total sample dry weight prior to digestion.

\section{Electron Balance Calculations}

COD was used to quantify electron availability and partitioning in gaseous, soluble, and particulate electron sinks. Theoretical COD values of substrates, $\mathrm{H}_{2}$, and PHB were calculated using Eq. 1 [20]:

$$
\begin{aligned}
\mathrm{C}_{n} \mathrm{H}_{a} \mathrm{O}_{b} \mathrm{~N}_{c} & +\left(\frac{2 n+0.5 a-1.5 c-b}{2}\right) \mathrm{O}_{2} \rightarrow n \mathrm{CO}_{2} \\
& +c \mathrm{NH}_{3}+\frac{a-3 c}{2} \mathrm{H}_{2} \mathrm{O}
\end{aligned}
$$

The stoichiometry in this reaction defines the theoretical oxygen demand for the full oxidation of an organic compound to $\mathrm{CO}_{2}$. For example, the moles of $\mathrm{O}_{2}$ required for the complete oxidation of $1 \mathrm{~mol}$ of lactic acid $\left(\mathrm{C}_{3} \mathrm{H}_{6} \mathrm{O}_{3}\right)$ can be calculated from Eq. 1 to be equal to $3 \mathrm{~mol}$ of $\mathrm{O}_{2}$. Since the molecular weight of $\mathrm{O}_{2}$ is $32 \mathrm{mg} / \mathrm{mmol}$, the theoretical COD of lactic acid is $96 \mathrm{mg}$ COD per millimole. Since the lactic acid experiments had an initial concentration of $39.5 \mathrm{mM}$, this corresponds to $3,800 \mathrm{mg}$ COD per liter. Glutamic acid $\left(\mathrm{C}_{5} \mathrm{H}_{9} \mathrm{O}_{4} \mathrm{~N}\right)$, the nitrogen source in all experiments, also contributes to the initial $\mathrm{COD}$ in the cultures. From Eq. 1 follows that $4.5 \mathrm{~mol} \mathrm{O}_{2}$ is required for the complete oxidation of $1 \mathrm{~mol}$ of glutamic acid. Thus, the theoretical COD of glutamic acid is $144 \mathrm{mg}$ COD per millimole, and since $8.1 \mathrm{mM}$ of glutamic acid was used in all experiments, this corresponds to approximately 1,200 mg COD per liter. Consequently, the targeted initial COD in the medium, for all experiments, was about $5,000 \mathrm{mg} / \mathrm{L}$. For PHB, a theoretical COD of $144 \mathrm{mg}$ COD per millimole of monomer $\left(\mathrm{C}_{4} \mathrm{H}_{6} \mathrm{O}_{2}\right)$ was calculated. For $\mathrm{H}_{2}$, the theoretical COD is $16 \mathrm{mg}$ COD per millimole.

For mixed or unknown organic material, the COD cannot be calculated from Eq. 1, but can be obtained experimentally. Thus, the COD of the biomass and culture supernatants were determined colorimetrically using $\mathrm{HACH}$ High Range (0-1,500 mg/L) COD kits (HACH Company, Loveland, CO). Standard curves were prepared with potassium phthalate, according to standard methods [27].

\section{Results}

To understand electron partitioning during growth and stationary phases in $R$. sphaeroides, we used sets of batch cultures incubated under identical conditions, with two or three cultures (i.e., biological replicates) sampled at different stages of incubation. Real-time monitoring of gas production and high-frequency sampling of cell growth were implemented in each batch culture. Individual growth curves were fit to a single Gompertz sigmoid function (cell density $=a(\exp (b(\exp (c t))))$ [28], where $a, b$, and $c$ are functional constants, and $t$ is time) by adjusting the time variable and finding a unique set of constants that defined a characteristic shape of the growth curve for the entire set of cultures. The best-fitting sigmoid curves were in good agreement with experimental data $\left(R^{2} \geq 0.99\right)$, which meant that individual growth profiles had similar shapes, and thus, the fitting could be used to synchronize these individual profiles within the same time frame, irrespective of the phase differences between cultures (i.e., because of variations in the lag phase). This procedure allowed the definition of five specific points in the growth curve in which electron partitioning was analyzed. These sampling points are illustrated in Fig. 2a, which shows growth and gas production results for a lactate-fed set of batch cultures. The first sampling point was at the onset of gas production 


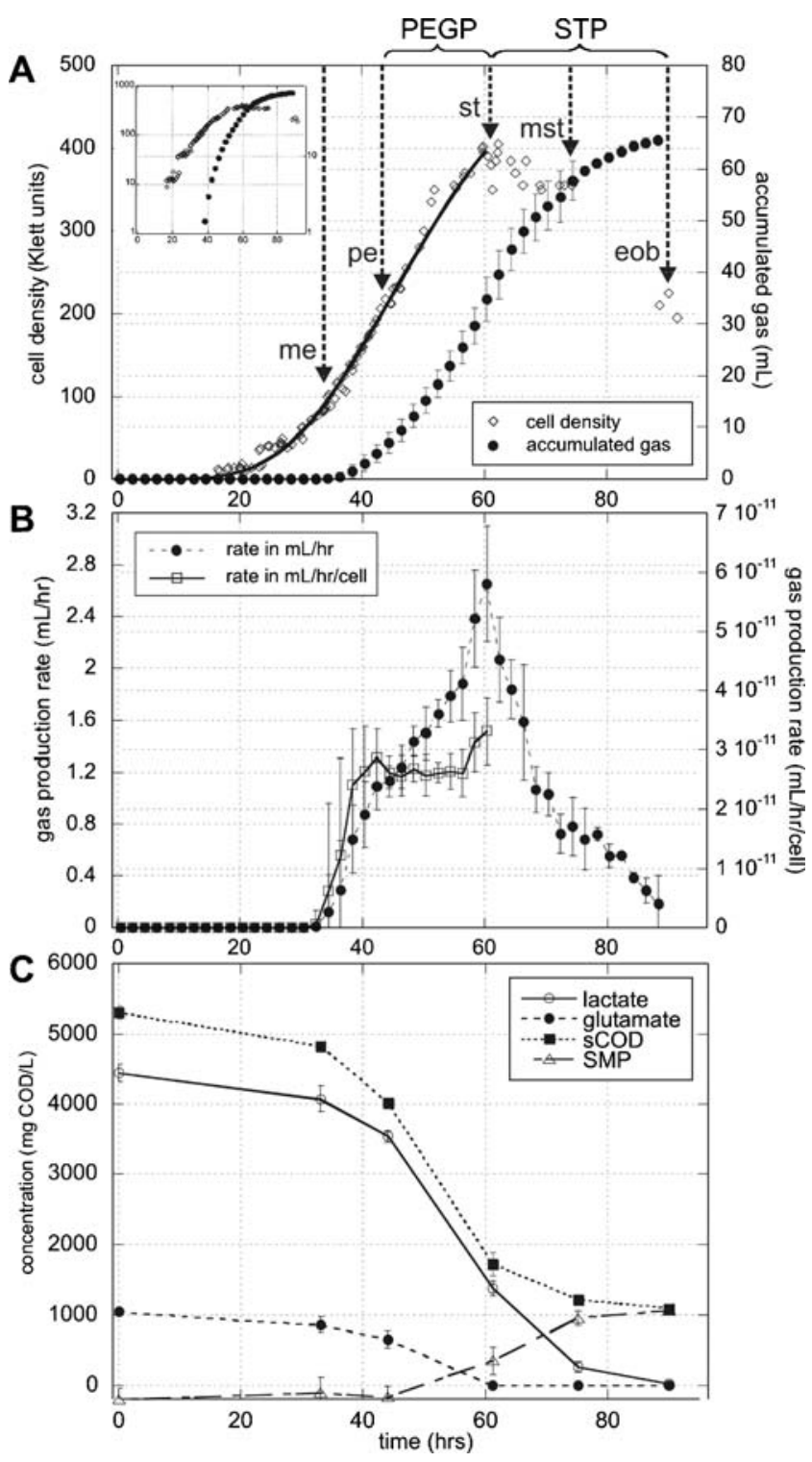

Fig. 2 Time series of batch cultures grown with lactate. a Gas production and absorbance values showing cell growth. Curve shows best-fitting sigmoid function (cell density $=594 \mathrm{exp}(-13.6 \mathrm{exp}$ $(-0.06 t)))$ which is used for aligning time frames of different batch cultures in the set. Sampling points are indicated by arrows. Regions of interest are indicated with horizontal brackets. Inset shows values in logarithmic scale. See also the list of abbreviations. b Gas production rates shown in milliliter per hour and in milliliter per hour per cell. Normalization by number of cells was based on the bestfitting figure in a, with Klett unit converted to cells according to 1 Klett $=10^{7}$ cells [22]. c Time series of lactate, glutamate, and total soluble compounds all expressed in milligram per liter oxygen demand. The difference of soluble COD $(s C O D)$ from the sum of lactate and glutamate gives the COD content of SMP. Error bars standard deviations

and coincided with midexponential growth (designated as me). The second was shortly after the transition from exponential to arithmetic growth and was defined as a postexponential (pe) sampling point. The third sampling point was at the beginning the stationary (st) phase and was characterized by having the maximum gas production rate (Fig. 2b). Samplings in the middle of the stationary (mst) phase and at the end of batch (eob), when gas production stopped, completed the set of sampling points.

At each of the sampled points, the concentrations of extracellular carbon and nitrogen sources were measured and converted to COD to quantify the reducing power remaining and consumed at different stages of growth. The COD profiles of the lactate-grown culture (Fig. 2c) indicated that about two thirds of lactate electrons had been utilized by the end of the growth phase (st point), and that the end of growth corresponded to the point where glutamate, the nitrogen source, was nondetectable. The remaining lactate was consumed by the cells during stationary phase. The difference between the measured soluble COD in the media and COD from the remaining carbon and nitrogen sources corresponded to SMP accumulation (see below for SMP analysis). In the case of lactate, SMP accumulation occurred mostly during stationary phase (Fig. 2c).

In addition to soluble $\mathrm{COD}, \mathrm{H}_{2}$ evolved from the culture and biomass accumulation was quantified at each sampling point. For the calculation of $\mathrm{COD}$ from $\mathrm{H}_{2}$, the gas composition was measured at regular intervals, and an average for the entire experiment was derived (e.g., $\mathrm{H}_{2}$ represented $87 \pm 3 \%$ of the evolved gas in lactate-fed cultures). The PHB component of the biomass was quantified separately, to complete COD calculations for all four major electron sinks depicted in Fig. 1. The resulting electron partitioning at each sampling point is illustrated in Fig. 3 for the lactate-fed culture. The COD sum of the end

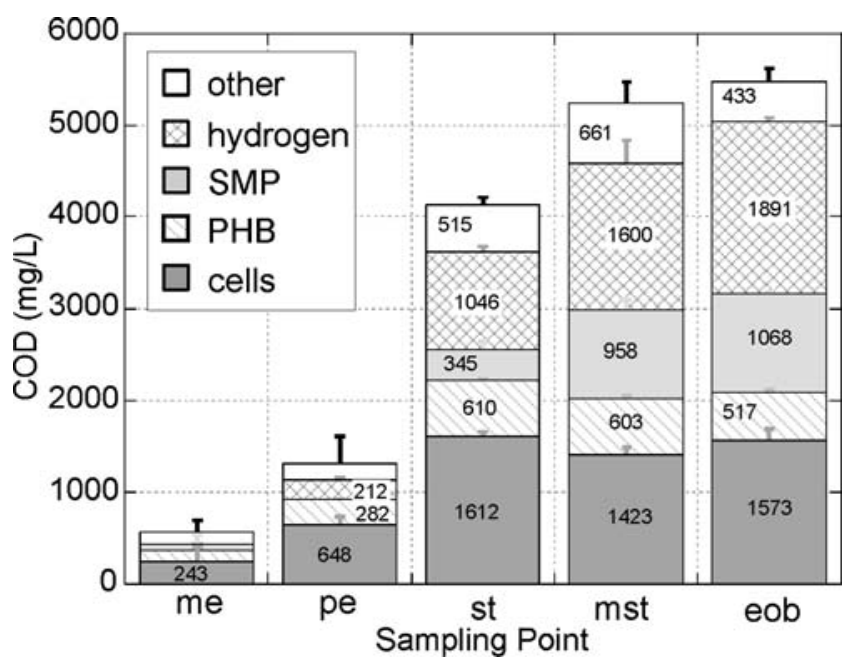

Fig. 3 Time series of COD distribution in lactate-fed batch cultures. Sampling points are indicated by abbreviations in Fig. 2a. Values greater than $200 \mathrm{mg} / \mathrm{L}$ are indicated on the bars. Error bars standard deviations. See also the list of abbreviations 
products is compared to the total substrate COD utilized (i.e., lactate and glutamate consumed) and the difference indicated as "COD ${ }_{\text {other }}$ " in Fig. 3, which represents potentially unaccounted electron sinks or the accumulation of experimental error from individual products. As shown in Fig. 3, the cumulative amount of electrons directed toward $\mathrm{H}_{2}$ production continuously increased, with $55 \%$ of the total produced during the growth phase and the rest in the stationary phase. Cell synthesis and PHB were important electron sinks only during the growth phase, while the importance of SMP in the electron balance significantly increased in the stationary phase. Furthermore, $\mathrm{COD}_{\text {other }}$ was less than $15 \%$ of the utilized COD for all time points, indicating that we can quantitatively account for most of the electrons derived from media components by measuring the products included in the study.

\section{Electron Partitioning with Different Carbon Sources}

Electron partitioning in cultures fed with different carbon sources are compared in Fig. 4, where COD values have been normalized dividing by the COD consumed at the end of each experiment. Since, in all cases, the total COD consumption was virtually equal to the sum of initial COD in glutamate and in the carbon source, the electron partitioning in Fig. 4 represents essentially all available electrons from the media components. The data shows that, in all cases, more than $85 \%$ of substrate electrons was partitioned into the known electron sinks (i.e., $\mathrm{f}_{\text {other }}<0.15$ in all cases). Succinate- and lactate-fed cultures had the highest partitioning into $\mathrm{H}_{2}$, while glucose-fed cultures

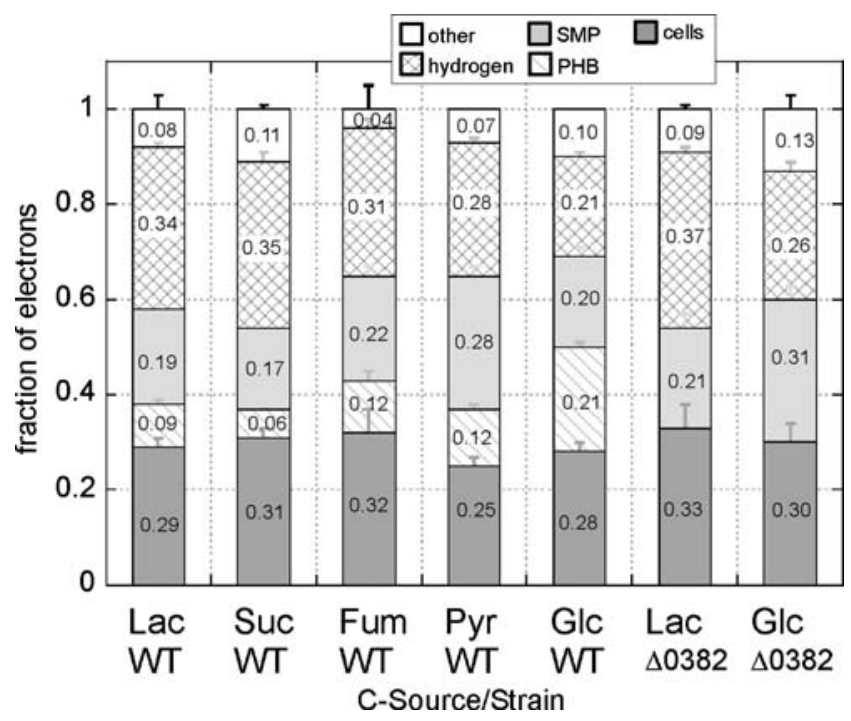

Fig. 4 Fate of substrate electrons for wild-type $(W T)$ and $\mathrm{PHB}(-)$ mutant $(\Delta 0382)$ cultures, calculated at the end of batch experiments with different organic substrates. Error bars standard deviations. See also the list of abbreviations produced significantly lower $\mathrm{H}_{2}$ than the organic acids tested. This trend was inversely correlated with PHB production, which was highest in glucose- and lowest for succinate- or lactate-fed cultures. An unexpected result was the significant fraction of electrons partitioning into SMP by the end of each experiment.

In addition to the end point analysis shown in Fig. 4, a comparison of electron fate during growth and stationary phases of the batch cultures is presented in Fig. 5. The phases are defined in Fig. 2a, as the postexponential growth phase (PEGP) and the stationary phase (STP). Notably, PEGP was characterized by a somewhat steady rate of $\mathrm{H}_{2}$ production at the single cell level, as the rate of gas evolution per cell and the percentage of $\mathrm{H}_{2}$ in the gas train did not vary significantly during this period (Fig. 2b). The electron partitioning in each phase was calculated by subtracting the COD values at the beginning point of each

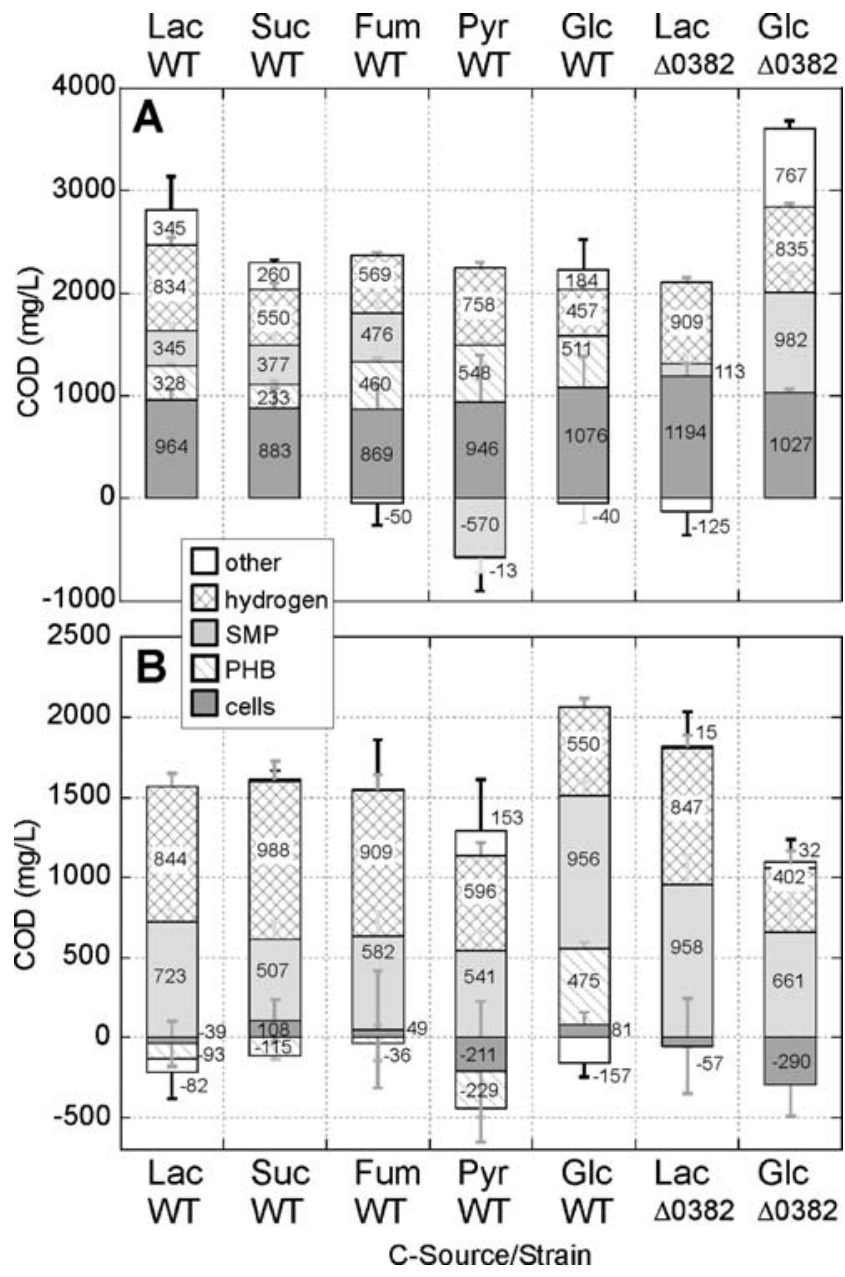

Fig. 5 COD distribution at the regions of interest shown in Fig. 2a. a Postexponential growth phase. b Stationary phase. Values greater than $10 \mathrm{mg}$ COD per liter are indicated on the bars. Error bars standard deviations calculated by error propagation. See also the list of abbreviations 
region from those at the end, and standard deviations were recalculated using standard error propagation techniques. During PEGP (Fig. 5a), biomass production was the main electron sink, followed by $\mathrm{H}_{2}$ and PHB production. During STP (Fig. 5b), biomass formation was generally negligible, and the main electron sinks were $\mathrm{H}_{2}$ and SMP. One exception to this generalization is the relatively large fraction of electrons accumulated in PHB by stationary phase cells in the glucose-fed culture (Fig. 5b), while in cultures fed with organic acids, net formation of PHB occurred only during growth (Fig. 5a, b). The negative values in Fig. 5 indicate product consumption rather than formation, and therefore, a general trend in the stationary phase was the utilization of small amounts of biomass and PHB as electron donors, except for the glucose culture, as explained above. Also, the net loss of SMP in PEGP by the pyruvate-fed culture (Fig. 5a) indicates a large production of SMP before the initiation of PEGP in this particular culture.

Table 1 provides a quantitative comparison of gas accumulation, composition, and production rates for all experiments. The biogas composition was similar in all cultures (average $\mathrm{H}_{2}$ content of $87 \pm 3 \%$ ), but the amount of gas accumulated and the gas production rates per cell depended highly on the substrate. When the fraction of electrons going to $\mathrm{H}_{2}$ at each phase is normalized with respect to the amount of electron donors consumed $\left(\mathrm{f}_{\mathrm{H} 2}\right.$ in Table 1), the organic acid-fed cultures show a higher utilization of $\mathrm{H}_{2}$ as electron sink in stationary phase than in the growth phase, but the glucose-fed culture shows by far the smallest partitioning of electrons into $\mathrm{H}_{2}$ in stationary phase, which correlates with the observed PHB accumulation during stationary phase in this culture. Thus, from the perspectives of maximizing $\mathrm{H}_{2}$ production, $\mathrm{PHB}$ formation seems to be an important competing electron sink during growth phase in all the cultures, but in the glucose-fed culture, PHB is also a competing electron sink during stationary phase.

\section{Electron Partitioning in $\mathrm{PHB}(-)$ Mutants}

Given the importance of PHB in the electron partitioning during growth and stationary phases, the effect of eliminating PHB as an electron sink was investigated with lactateor glucose-fed cultures using electron balances for the overall batch (Fig. 4) and separately analyzing electron partitioning in PEGP and STP (Fig. 5). Overall electron balances in the $\mathrm{PHB}(-)$ mutant (Fig. 4) showed that electrons were redistributed among several other products, including $\mathrm{H}_{2}$, SMP, and cells. In the lactate-fed $\mathrm{PHB}(-)$ mutant culture, the increase in the electron share of $\mathrm{H}_{2}$ (from $34 \%$ to $37 \%$ ) was small but significant at $p=0.05$ (based on a Student's $t$ test) and reproducible in independent experiments with lactate (data not shown), whereas the gain in electrons partitioning to $\mathrm{H}_{2}$ in the $\mathrm{PHB}(-)$ mutant was comparatively larger for glucose-fed cells $(21 \%$ to $26 \%$ ) with a $p=0.01$, consistent with the electron partitioning to PHB being larger with glucose-fed wild type cells.

\section{Analysis of SMP}

Since the SMP pool was significant in all the cultures, we further analyzed SMP in two ways. Firstly, we quantified the overall size profile of the COD represented in SMP by filtering the soluble portion of cultures through 3- and $10-k D a$ filters. Secondly, we identified and quantified the low molecular weight, extracellular organic acids during PEGP and STP (Fig. 6).

In general, there was significant variability in the size distribution of SMP between batch experiments with

Table 1 Key parameters describing the performance of batch cultures

\begin{tabular}{|c|c|c|c|c|c|c|c|c|}
\hline & Growth phase & $\begin{array}{l}\text { Lactate } \\
\text { WT }\end{array}$ & $\begin{array}{l}\text { Succinate } \\
\text { WT }\end{array}$ & $\begin{array}{l}\text { Fumarate } \\
\text { WT }\end{array}$ & $\begin{array}{l}\text { Pyruvate } \\
\text { WT }\end{array}$ & $\begin{array}{l}\text { Glucose } \\
\text { WT }\end{array}$ & $\begin{array}{l}\text { Lactate } \\
\Delta 0382\end{array}$ & $\begin{array}{l}\text { Glucose } \\
\Delta 0382\end{array}$ \\
\hline \multirow[t]{3}{*}{ Gas produced $(\mathrm{mL})$} & Overall & $67 \pm 1$ & $61 \pm 4$ & $56 \pm 3$ & $51 \pm 2$ & $40 \pm 2$ & $72 \pm 3$ & $52 \pm 3$ \\
\hline & PEGP & $30 \pm 2$ & $20 \pm 2$ & $21 \pm 1$ & $28 \pm 2$ & $18 \pm 1$ & $33 \pm 1$ & $32 \pm 1$ \\
\hline & STP & $32 \pm 2$ & $35 \pm 4$ & $33 \pm 3$ & $23 \pm 2$ & $21 \pm 2$ & $32 \pm 3$ & $17 \pm 3$ \\
\hline $\begin{array}{l}\text { Average gas production } \\
\text { rate }\left(\mathrm{mL} / \mathrm{h} / 10^{11} \text { cells }\right)^{\mathrm{a}}\end{array}$ & PEGP & $2.7 \pm 0.3$ & $2.9 \pm 0.1$ & $2.3 \pm 0.2$ & $1.5 \pm 0.2$ & $1.4 \pm 0.2$ & $3.2 \pm 0.6$ & $2.8 \pm 0.3$ \\
\hline $\begin{array}{l}\text { Average hydrogen } \\
\text { content (\% gas) }\end{array}$ & Overall & $87 \pm 3$ & $90 \pm 3$ & $90 \pm 4$ & $85 \pm 1$ & $83 \pm 4$ & $88 \pm 2$ & $82 \pm 4$ \\
\hline \multirow[t]{2}{*}{$f_{\mathrm{H} 2}{ }^{b}$} & PEGP & $0.30 \pm 0.00$ & $0.24 \pm 0.01$ & $0.24 \pm 0.01$ & $0.45 \pm 0.02$ & $0.21 \pm 0.00$ & $0.43 \pm 0.01$ & $0.23 \pm 0.01$ \\
\hline & STP & $0.62 \pm 0.01$ & $0.66 \pm 0.02$ & $0.60 \pm 0.02$ & $0.70 \pm 0.03$ & $0.29 \pm 0.02$ & $0.48 \pm 0.03$ & $0.50 \pm 0.02$ \\
\hline
\end{tabular}

\footnotetext{
${ }^{\text {a }}$ Gas production rate per cell was calculated using time intervals of $20 \mathrm{~min}$ and the cell numbers derived from the best-fitting growth curves at the corresponding time points. An average was calculated over PEGP

${ }^{b}$ Fraction of substrate electrons partitioning into $\mathrm{H}_{2}$, defined as $\mathrm{COD}_{\mathrm{H} 2} / \mathrm{COD}_{\text {utilized }}$
} 


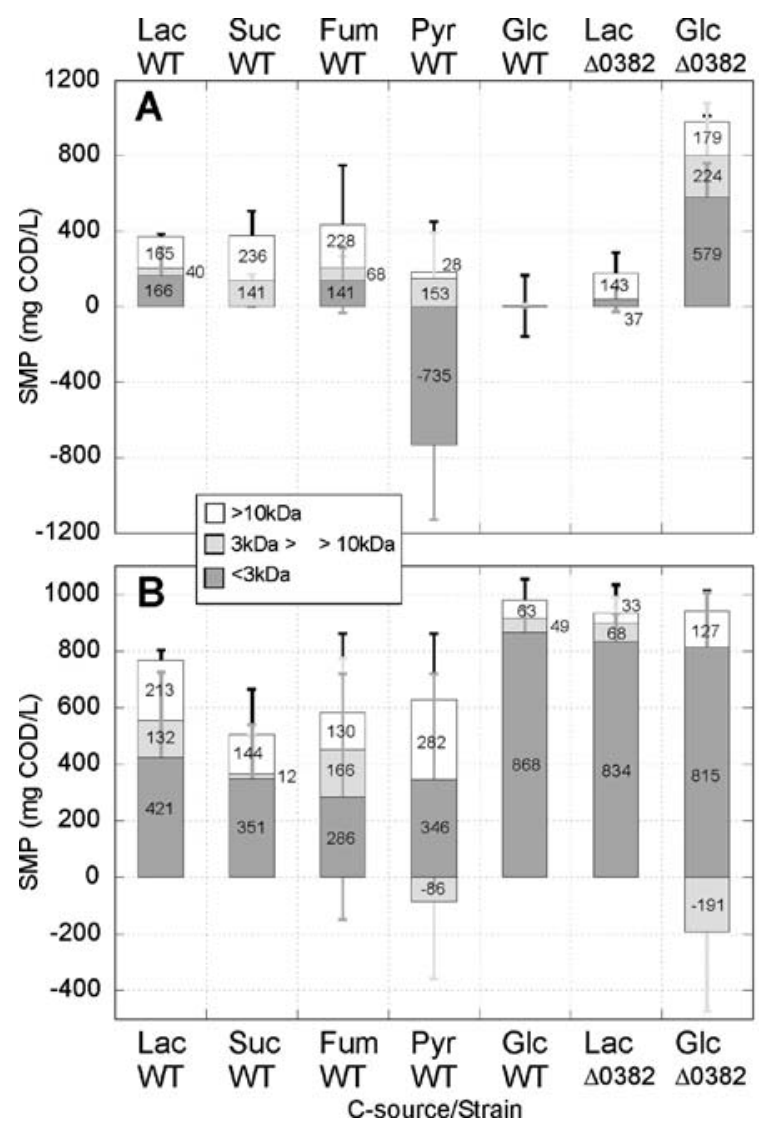

Fig. 6 Analysis of SMP formed and/or consumed during the batch tests. Left panel Size distribution analysis of SMP in postexponential growth phase (a) and stationary phase (b). Right panel Quantification

different carbon sources (Fig. 6). The data indicate that the SMP produced during STP was mostly $<3-\mathrm{kDa}$ molecules (Fig. 6b), while the size distribution was more dispersed during the PEGP (Fig. 6a). Macromolecules larger than $10 \mathrm{kDa}$ were produced by most cultures during both growth phases and always showed net average production, suggesting little or no degradation of this SMP class. However, smaller size SMP was both produced and consumed.

SMP identified in the $<3-\mathrm{kDa}$ fraction included tricarboxylic acid (TCA) cycle intermediates, pyruvate and lactate (Fig. 6c, d). These generally did not make a large fraction of the $<3-\mathrm{kDa}$ portion, except in the glucose-fed culture of the $\mathrm{PHB}(-)$, in which, pyruvate was a major product during PEGP and was completely consumed during STP. For the other cultures, the $<3-\mathrm{kDa}$ SMP mostly consisted of unknown molecules, although moderate amounts of pyruvate, malate, and lactate accumulated in PEGP and were consumed in STP. The large amount of SMP consumed by the growing pyruvate culture and characterized as $<3 \mathrm{kDa}$ (Fig. 6a) remained largely unidentified, as there was no consumption of identified molecules during PEGP. Finally, succinate emerged as a common SMP produced during STP, although in moderate amounts (Fig. 6d).

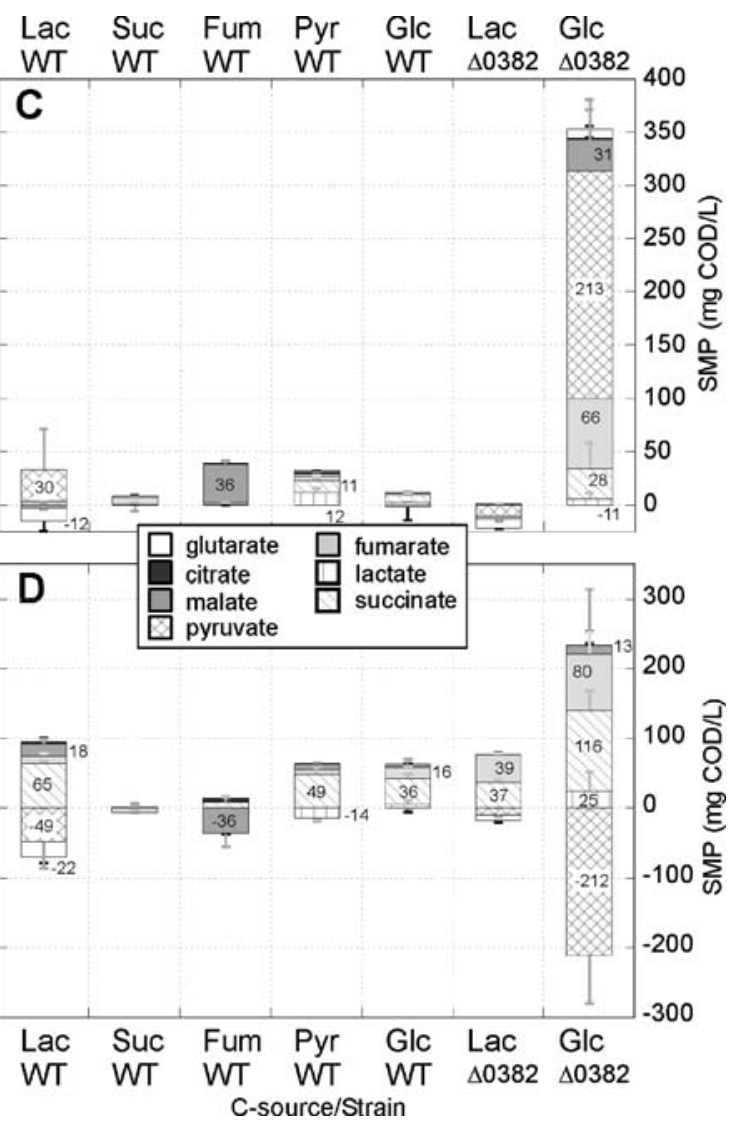

of identified organic acids in postexponential growth phase (c) and stationary phase (d). Error bars standard deviations calculated by error propagation. See also the list of abbreviations

\section{Discussion}

While photosynthetic microbes are among those being considered for biofuel production, little is known about the fate of electrons derived from light- or nutrient-driven metabolic processes. Therefore, it is desirable to develop approaches to quantify the amount of reducing power that is directed into fuels and major microbial end products and to use this information to increase the production of biofuels. In this study, we applied COD-based approaches to quantify electron fate during $\mathrm{H}_{2}$ production by the photosynthetic microbe $R$. sphaeroides. However, the assays and approaches described in this paper are applicable to other types of biofuel production by this and other microbes provided that electron donors and acceptors are either directly converted to COD units, or indirectly quantified in COD units by measuring products of their reactions.

During anoxic growth of photoheterotrophic organisms, reduced compounds present in the growth medium are the major source of electrons, as light energy capture is a cyclic process with no net electron gain [29]. Thus, by measuring the gaseous, liquid, and solid products of photoheterotro- 
phic activity (Fig. 1), we were able to calculate an overall mass balance of electrons after converting the measurements to the common unit of COD. The unaccounted proportion of electrons (i.e., $f_{\text {other }}$ in Fig. 1) was generally $0-15 \%$, indicating that the electron sinks considered in our conceptual model were the major ones.

\section{Comparison of $\mathrm{H}_{2}$ Production by Growing and Resting Cells}

$\mathrm{H}_{2}$ production by cultures of photoheterotrophic bacteria has been explored using resting or immobilized cell cultures $[14,30-32]$ to eliminate biomass formation as a competing reaction. However, to develop continuous flow $\mathrm{H}_{2}$-producing bioreactors [33], a growing culture is needed so biomass is a significant competing electron sink. By comparing electron partitioning analysis during growth and stationary phases (i.e., PEGP and STP regions defined in Fig. 2), our analysis provides a direct comparison of biofuel production efficiency during each stage (Fig. 5). It is evident from our results that the efficiency of fuel production depends not only on the growth conditions, but also on the ultimate aim of the process to be engineered. For instance, although the highest rate of $\mathrm{H}_{2}$ production is achieved at the end of exponential growth (Fig. 2b), electron partitioning into this fuel is still relatively low at this stage (Table 1) since biomass production is a major competitor. In contrast, the fraction of electrons routed to $\mathrm{H}_{2}$ is higher in stationary phase, while production rates follow a decreasing trend as one or more nutrients becomes limiting. Thus, if the goal is to maximize $\mathrm{H}_{2}$ production rates, flow-through bioreactors with a capacity for high cell concentrations might be desirable, even though light penetration might be a concern with photosynthetic microbes [33, 34]. However, if the goal is to maximize the conversion of one or more renewable substrates to $\mathrm{H}_{2}$, immobilized nongrowing cultures are predicted to be optimal [30, 32]. Finally, if the aim is to couple $\mathrm{H}_{2}$ production with either waste treatment [10] or removal of organic substrates from a liquid stream [35], then efficient substrate consumption in a plug flow reactor might provide the best results [33]. In that case, the electron balance of the entire experiment, including growth and stationary phases, may be a better guide for process design, although the discovery that a significant fraction of electrons can be partitioned to SMP suggests a limit in the removal efficiency of organic substrates.

Correlations of $\mathrm{H}_{2}$ and PHB Production with Free Energy of Organic Substrates

Analysis of our data allows two noteworthy correlations regarding electron partitioning in various cultures, which could potentially lead to a new approach for predicting $\mathrm{H}_{2}$ and PHB formation in photoheterotrophic cultures as a function of the organic substrate. Firstly, the fraction of electrons partitioned into $\mathrm{H}_{2}$ (Fig. 4) inversely correlate with the free energy changes of the electron donor halfreactions, on a per electron equivalent basis $(r=-0.97, p<$ 0.01 ; Fig. 7). These free energy values were calculated based on free energies of formation of individual species [36] for a stoichiometrically balanced half-reaction normalized per unit of electrons transferred [20]. The correlation suggests that electron partitioning into $\mathrm{H}_{2}$ could be predicted by the free energy change in the degradation of the substrate to a common oxidized intermediate, rather than the initial oxidation state of the substrate. Indeed, while lactate and glucose have the same oxidation state, they were on the opposite ends of the $\mathrm{H}_{2}$ production spectrum. Secondly, the fraction of electrons going to PHB is also related to free energy changes of the substrate halfreactions (Fig. 7), in this case with a positive correlation $(\mathrm{r}=0.99, p<0.01)$. Interestingly, the sum of $\mathrm{f}_{\mathrm{H} 2}$ and $\mathrm{f}_{\mathrm{PHB}}$ was nearly constant $(0.42 \pm 0.02)$ over the five different carbon sources, as can be inferred from the slopes of $\mathrm{H}_{2}$ and PHB lines, whose absolute values are almost identical (Fig. 7). Thus, the inverse relationship in Fig. 7 clearly shows the competing nature of $\mathrm{PHB}$ and $\mathrm{H}_{2}$ formation for substrate electrons, although the mechanisms behind this competition and the free energy relationships are not clear. In contrast, there was no obvious correlation between SMP production and substrate free energy $(r=0.25, p>0.65)$,

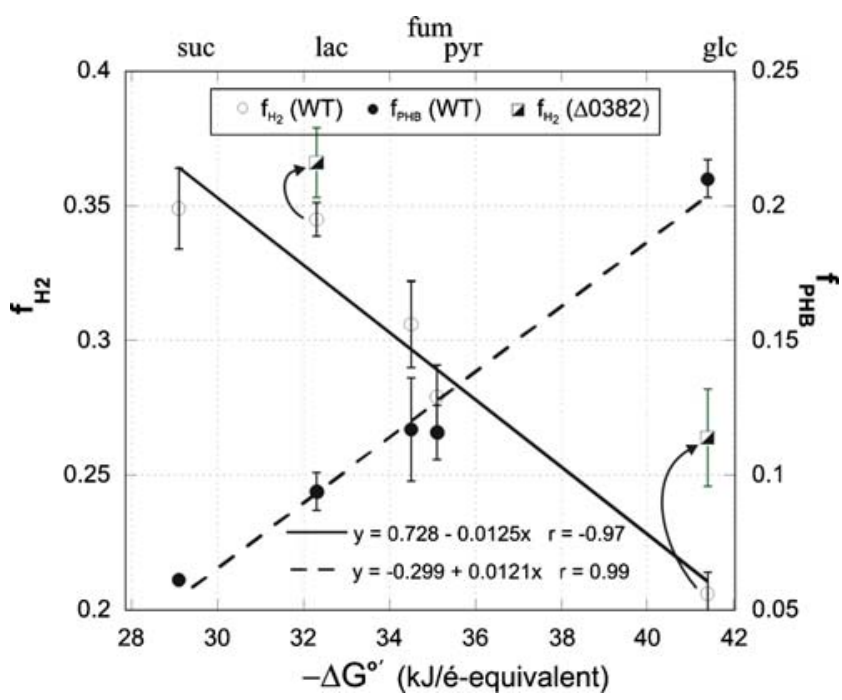

Fig. 7 Correlation of free energy changes of electron donor halfreactions with electron partitioning into $\mathrm{H}_{2}$ and PHB. Free energy values are calculated for $\mathrm{pH}=7$ and normalized to one-electron reactions [20]. Arrows show the increase in electron partitioning into $\mathrm{H}_{2}$ upon PHB mutation. The positions of carbon sources on the free energy line are indicated on the upper axis. Error bars standard deviations. See also the list of abbreviations 
possibly reflecting the diverse chemical nature of the SMP pool observed with different organic substrates (Fig. 6).

Effects of Genetic Changes on the Distribution of Electrons

The strategy employed in this study is also suited for testing the predictions on the effects of removing individual electron sinks on production of $\mathrm{H}_{2}$ or other candidate fuel molecules. While PHB mutants of photoheterotrophic organisms have been used for increased $\mathrm{H}_{2}$ production before [37-40], little is known about how cells balance electron flux in these or other mutant strains. Our data indicate that loss of PHB synthesis removed a significant electron sink. However, the magnitude of the effect of blocking PHB synthesis on biofuel production depended on the substrate being utilized. For lactate, the net gain in $\mathrm{H}_{2}$ production by the PHB mutation was $7 \%$, compared to $\mathrm{H}_{2}$ production in wild-type cultures (Table 1). Overall, the electron flux that was deviated from PHB was captured in $\mathrm{H}_{2}$ production ( $33 \%$ of electron flux), cell synthesis (44\%), and SMP (22\%). For glucose, there was a $30 \%$ higher $\mathrm{H}_{2}$ production in the $\mathrm{PHB}(-)$ mutant (Table 1). However, only $24 \%$ of the electron flux originally going to PHB in the wild-type cells contributed to increased $\mathrm{H}_{2}$ production in the $\mathrm{PHB}(-)$ mutant, as SMP was the most significant electron sink, receiving $52 \%$ of the deviated electron flux. The remaining flux corresponded to cell synthesis $(9.5 \%)$ and an increase in the unaccounted $\mathrm{COD}_{\text {other }}$ fraction.

Electron partitioning of the $\mathrm{PHB}(-)$ mutant was also analyzed during PEGP and STP (Fig. 5). In the lactate-fed $\mathrm{PHB}(-)$ mutant culture, the absolute values of $\mathrm{H}_{2} \mathrm{COD}$, as compared to those of the wild type, did not reflect the elimination of PHB production during growth and lack of electrons from PHB degradation during stationary phase (Fig. 5). This can be explained by considering the different amounts of total COD utilized during PEGP and STP in the cultures. When the total COD was used to normalize data, the fraction of $\mathrm{H}_{2}$ electrons in $\mathrm{PHB}(-)$ mutant was more than that in wild type during PEGP and less during STP (Table 1), as expected.

In the growth phase of the glucose-fed mutant cultures, there were approximately twofold increases in gas production rate and in $\mathrm{H}_{2}$ accumulation compared to glucose-fed wild-type cultures (Fig. 5a, Table 1). Accordingly, the fraction of glucose utilized during PEGP by the $\mathrm{PHB}(-)$ mutant increased significantly. During the stationary phase, the $\mathrm{PHB}(-)$ mutant yielded less $\mathrm{H}_{2}$ (Fig. 5b), despite the fact that $\mathrm{PHB}$ formation was a significant competing electron sink for the wild-type culture in this phase (Fig. 5b). This decrease is consistent with substrate utilization during growth, as less glucose was available at the initiation of the stationary phase in the $\mathrm{PHB}(-)$ mutant culture.
The increased $\mathrm{H}_{2}$ production in the $\mathrm{PHB}(-)$ mutant cultures was comparable to $\mathrm{H}_{2}$ production by wild-type cultures fed with pyruvate or fumarate (Table 1). This is important since biofuel production processes might use mixed organic acids for photoheterotrophic $\mathrm{H}_{2}$ production, following a dark fermentation step [9-11]. In this case, the benefit of utilizing a $\mathrm{PHB}(-)$ mutant would be small, since $\mathrm{H}_{2}$ production by wild-type cells is already high when fed organic acid substrates. On the contrary, the benefit of eliminating PHB accumulation would be more important during direct photoheterotrophic conversion of sugars to $\mathrm{H}_{2}$, because of the lower $\mathrm{H}_{2}$ production from sugars by the wild-type cells.

\section{SMP as a Major Electron Sink}

A surprising finding in this study was the observation that SMP can be a major electron sink, thus taking a large amount of reducing power away from biofuel production, especially in resting cells. SMP accumulation was shown to be considerably larger than PHB formation, and therefore, a logical target for further attempts to optimize $\mathrm{H}_{2}$ production would be the control of genetic and metabolic systems that lead to SMP production. Under the conditions described in this study, only a small fraction of the SMP corresponded to common metabolites such as lactate and TCA cycle intermediates (Fig. 6), and most remained unidentified low or high $(>3 \mathrm{kDa})$ molecular weight material. In general, there is a fundamental lack of knowledge regarding SMP formation during photoheterotrophic growth, and in addition to the products identified in this study, formate is the only other organic acid that has been described as a byproduct of $R$. sphaeroides photoheterotrophic metabolism [33, 41], although this compound was not detected in any of the cultures in this study.

It is possible that the large fraction of high-molecular weight unidentified SMP could be extracellular polymeric substances (EPS), which are known to play a major role in cell aggregation $[42,43]$. Although cell aggregation did not seem to occur to a significant extent in growing and early stationary phase cultures, there was noticeable cell sedimentation in late stationary phase, consistent with the possibility of EPS formation. Thus, the $>10-\mathrm{kDa}$ SMP observed in the cultures could possibly correspond to EPS precursors, a hypothesis that requires further investigation.

\section{Conclusions}

In conclusion, a COD-based method was developed and used for the quantitative assessment of electron partitioning in $\mathrm{H}_{2}$-producing $R$. sphaeroides cultures. This approach is useful for evaluating biofuel production by wild-type and 
mutant cultures in both growing and resting cells. The results with selected organic substrates revealed correlations of $\mathrm{H}_{2}$ production and PHB accumulation with the free energy for oxidation of the substrate, normalized on an electron equivalent basis. We also showed that the method is applicable to the analysis of the redistribution of reducing power, when a known electron sink, PHB synthesis, is removed by genetic modification. SMP was a significant electron sink competing with biofuel production, so future studies should target identification and removal of the pathways that will divert electrons from $\mathrm{H}_{2}$ or other desired biofuel products to SMP. Finally, the data generated on the distribution of reducing power for growing and resting cells can be useful to inform genome-scale metabolic network models of $R$. sphaeroides that will provide predictions on the flow of reducing power needed to further improve biofuel production.

Acknowledgments This research was supported by the Office of Science, Department of Energy, under grants DE-FG02-07ER64495 and DE-FC02-07ER64494. We are indebted to Jackie B. Cooper for her help with analytical equipment and to Rodolfo Perez for valuable discussions and technical help. We also thank Yasar Yilmaz for constructing the light box used in this study.

\section{References}

1. Demirbas A (2008) Biofuels sources, biofuel policy, biofuel economy and global biofuel projections. Energy Convers Manage 49:2106-2116

2. US Department of Energy (2008) A decade of discovery. http:// www.energy.gov/discovery/

3. Mueller-Langer F, Tzimas E, Kaltschmitt M, Peteves S (2007) Techno-economic assessment of hydrogen production processes for the hydrogen economy for the short and medium term. Int $\mathrm{J}$ Hydrogen Energy 32:3797-3810

4. Das D, Veziroglu TN (2001) Hydrogen production by biological processes: a survey of literature. Int J Hydrogen Energy 26:13-28

5. Hallenbeck PC, Ghosh D (2009) Advances in fermentative biohydrogen production: the way forward? Trends in Biotechnology 27:287-297

6. Gest H, Kamen M (1949) Photoproduction of molecular hydrogen by Rhodospirillum rubrum. Science 109:558-559

7. Kapdan IK, Kargi F (2006) Bio-hydrogen production from waste materials. Enzyme Microb Technol 38:569-582

8. Rey FE, Heiniger EK, Harwood CS (2007) Redirection of metabolism for biological hydrogen production. Appl Environ Microbiol 73:1665-1671

9. Chen CY, Yang MH, Yeh KL, Liu CH, Chang JS (2008) Biohydrogen production using sequential two-stage dark and photo fermentation processes. Int J Hydrogen Energy 33:47554762

10. Eroglu E, Eroglu I, Gunduz U, Turker L, Yucel M (2006) Biological hydrogen production from olive mill wastewater with two-stage processes. Int J Hydrogen Energy 31:1527-1535

11. Nath K, Kumar A, Das D (2005) Hydrogen production by Rhodobacter sphaeroides strain OU001 using spent media of Enterobacter cloacae strain DM11. Appl Microbiol Biotechnol 68:533-541
12. Fascetti E, Todini O (1995) Rhodobacter sphaeroides RV cultivation and hydrogen production in a one- and two-stage chemostat. Appl Microbiol Biotechnol 44:300-305

13. Koku H, Eroglu I, Gunduz U, Yucel M, Turker L (2003) Kinetics of biological hydrogen production by the photosynthetic bacterium Rhodobacter sphaeroides OU 001. Int J Hydrogen Energy 28:381388

14. Zhu HG, Suzuki T, Tsygankov AA, Asada Y, Miyake J (1999) Hydrogen production from tofu wastewater by Rhodobacter sphaeroides immobilized in agar gels. Int J Hydrogen Energy 24:305-310

15. Cho YK, Donohue TJ, Tejedor I, Anderson MA, McMahon KD, Noguera DR (2008) Development of a solar-powered microbial fuel cell. J Appl Microbiol 104:640-650

16. Callister SJ, Dominguez MA, Nicora CD, Zeng XH, Tavano CL, Kaplan S et al (2006) Application of the accurate mass and time tag approach to the proteome analysis of sub-cellular fractions obtained from Rhodobacter sphaeroides 2.4.1. aerobic and photosynthetic cell cultures. Journal of Proteome Research 5:1940-1947

17. Donohue TJ, Kaplan S (1991) Genetic techniques in Rhodospirillaceae. Methods Enzymol 204:459-485

18. Mackenzie C, Choudhary M, Larimer FW, Predki PF, Stilwagen S, Armitage JP et al (2001) The home stretch, a first analysis of the nearly completed genome of Rhodobacter sphaeroides 2.4.1. Photosynth Res 70:19-41

19. Koku H, Eroglu I, Gunduz U, Yucel M, Turker L (2002) Aspects of the metabolism of hydrogen production by Rhodobacter sphaeroides. Int J Hydrogen Energy 27:1315-1329

20. Rittmann BE, McCarty PL (2001) Environmental biotechnology: principles and applications. McGraw-Hill, Boston

21. Sistrom W (1960) A requirement for sodium in the growth of Rhodopseudomonas sphaeroides. J Gen Microbiol 22:778-785

22. Tai SP, Kaplan S (1985) Intracellular localization of phospholipid transfer activity in Rhodopseudomonas sphaeroides and a possible role in membrane biogenesis. J Bacteriol 164:181-186

23. Villas-Boas SG, Delicado DG, Akesson M, Nielsen J (2003) Simultaneous analysis of amino and nonamino organic acids as methyl chloroformate derivatives using gas chromatography-mass spectrometry. Anal Biochem 322:134-138

24. Siluiter A, Hames B, Ruiz R, Scarlata C, Sluiter J, Templeton D (2006) Determination of sugars, byproducts, and degradation products in liquid fraction process samples, vol. Technical Report: NREL/TP-520-42623. National Renewable Energy Laboratory

25. Comeau Y, Hall KJ, Oldham WK (1988) Determination of polybeta-hydroxybutyrate and poly-beta-hydroxyvalerate in activatedsludge by gas-liquid chromatography. Appl Environ Microbiol 54:2325-2327

26. Drnevich MA, Noguera DR (2004) Production of polyhydroxyalkanoate during treatment of low-phosphorus-content wastewater. Water Environ Res 76:380-383

27. American Public Health Association (1989) 5220D Closed Reflux, calorimetric method. In: Clesceri LS, Greenberg AE, Trussell RR (eds) Standard methods for the examination of water and wastewater, 17th edn. American Public Health Association, Washington, DC, pp 5-15-5-16

28. Gompertz B (1825) On the nature of the function expressive of the law of human mortality, and on a new mode of determining the value of life contingencies. Phil Trans Roy Soc London 115:513-585

29. Johnson ET, Schmidt-Dannert C (2008) Light-energy conversion in engineered microorganisms. Trends in Biotechnology 26:682689

30. Gosse JL, Engel BJ, Rey FE, Harwood CS, Scriven LE, Flickinger MC (2007) Hydrogen production by photoreactive nanoporous latex coatings of nongrowing Rhodopseudomonas palustris CGA009. Biotechnology Progress 23:124-130 
31. Hillmer P, Gest H (1977) H-2 metabolism in photosynthetic bacterium Rhodopseudomonas capsulata-production and utilization of H-2 by resting cells. J Bacteriol 129:732-739

32. Melnicki MR, Bianchi L, De Philippis R, Melis A (2008) Hydrogen production during stationary phase in purple photosynthetic bacteria. Int J Hydrogen Energy 33:6525-6534

33. Eroglu I, Tabanoglu A, Gunduz U, Eroglu E, Yucel M (2008) Hydrogen production by Rhodobacter sphaeroides OU001 in a flat plate solar bioreactor. Int J Hydrogen Energy 33:531-541

34. Basak N, Das D (2009) Photofermentative hydrogen production using purple non-sulfur bacteria Rhodobacter sphaeroides OU001 in an annular photobioreactor: a case study. Biomass Bioenergy 33:911-919

35. Kobayashi M, Kobayashi M (1995) Remediation and treatment using anoxygenic phototrophic bacteria. In: Blankenship RE, Madigan MT, Bauer CE (eds) Anoxygenic photosynthetic bacteria. Kluwer Academic Publishers Dordrecht, Netherlands, pp 1269-1282

36. Thauer RK, Jungermann K, Decker K (1977) Energy conservation in chemotropic anaerobic bacteria. Bacteriol Rev 41:100-180

37. Franchi E, Tosi C, Scolla G, Della Penna G, Rodriguez F, Pedroni PM (2004) Metabolically engineered Rhodobacter sphaeroides RV strains for improved biohydrogen photoproduction combined with disposal of food wastes. Mar Biotechnol 6:552-565
38. Hustede E, Steinbuchel A, Schlegel HG (1993) Relationship between the photoproduction of hydrogen and the accumulation of $\mathrm{Phb}$ in nonsulfur purple bacteria. Appl Microbiol Biotechnol 39:87-93

39. Kim MS, Baek JS, Lee JK (2006) Comparison of H-2 accumulation by Rhodobacter sphaeroides KD131 and its uptake hydrogenase and PHB synthase deficient mutant. Int J Hydrogen Energy 31:121-127

40. Lee IH, Park JY, Kho DH, Kim MS, Lee JK (2002) Reductive effect of $\mathrm{H}-2$ uptake and poly-beta-hydroxybutyrate formation on nitrogenase-mediated $\mathrm{H}-2$ accumulation of Rhodobacter sphaeroides according to light intensity. Appl Microbiol Biotechnol 60:147153

41. Zabut B, Ei-Kahlout K, Yucel M, Gunduz U, Turker L, Eroglu I (2006) Hydrogen gas production by combined systems of Rhodobacter sphaeroides OU001 and Halobacterium salinarum in a photobioreactor. Int J Hydrogen Energy 31:1553-1562

42. Sheng GP, Yu HQ, Yu Z (2005) Extraction of extracellular polymeric substances from the photosynthetic bacterium Rhodopseudomonas acidophila. Appl Microbiol Biotechnol 67:125-130

43. Watanabe M, Sasaki K, Nakashimada Y, Kakizono T, Noparatnaraporn N, Nishio N (1998) Growth and flocculation of a marine photosynthetic bacterium Rhodovulum sp. Appl Microbiol Biotechnol 50:682-691 